\title{
Passive Flow Control Around a Semi-Circular Cylinder Using Porous Coatings
}

\author{
Chloé Mimeau \\ Univ. Grenoble-Alpes, LJK \\ 51 , rue des Mathématiques \\ F-38041 Grenoble FRANCE \\ Email: Chloe.Mimeau@imag.fr
}

\author{
Iraj Mortazavi \\ IMB Université de Bordeaux \\ $M C^{2}$ INRIA Bordeaux Sud-Ouest \\ 351, cours de la Libération \\ F-33405 Talence FRANCE \\ Email: Iraj.Mortazavi@math.u-bordeaux1.fr
}

\author{
Georges-Henri Cottet \\ Univ. Grenoble-Alpes, LJK \\ 51, rue des Mathématiques \\ F-38041 Grenoble FRANCE \\ Email: Georges-Henri.Cottet@imag.fr
}

\begin{abstract}
In this paper, the passive control of flow past a semi-circular cylinder is investigated. This control is achieved by adding a porous medium between the solid obstacle and the incompressible fluid in order to reduce drag forces and regularize the flow. A vortex-penalization method is chosen to easily model the flow in the different media. Several configurations of the porous layer are investigated and parametric studies are performed in order to determine the most efficient passive flow control devices. This control study can be considered as a first step to propose efficient strategies to regularize the flow around a side-view mirror.
\end{abstract}

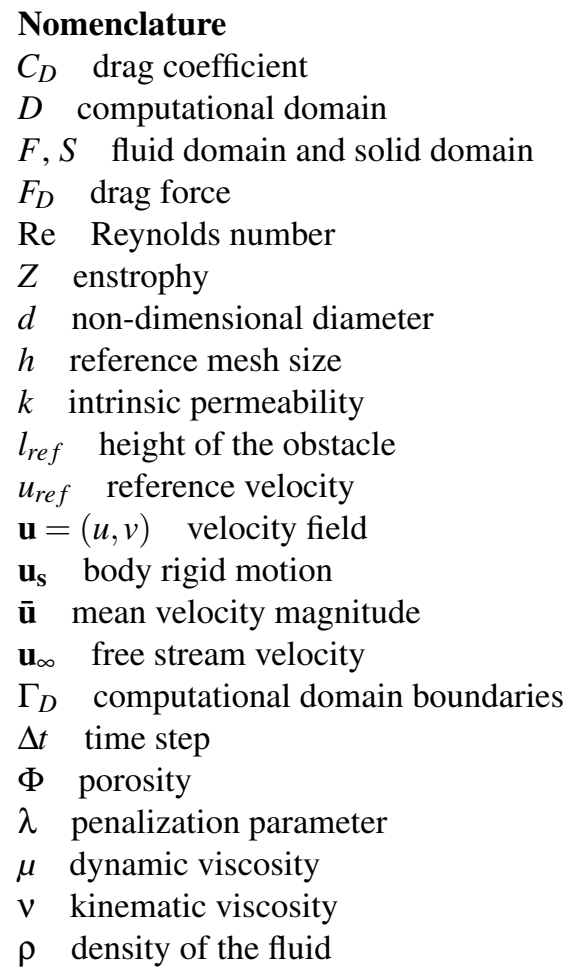


$\tau$ porous layer thickness

$\chi_{S}$ characteristic function

$\omega$ vorticity field

\section{Introduction}

On a ground vehicle, the outside mirrors, due to their spanwise position, indeed generate a non-negligible wake which interferes with the flow past car sides. They are responsible of up to $10 \%$ of the total vehicle drag but they only represent $0.5 \%$ of the total projected surface, which accounts for a good motivation to perform flow control past these obstacles. This work is devoted to the control of flow past a two-dimensional semi-circular cylinder which can be considered as a simplified section of a side-view mirror. As it was shown in [1,2], a flow past a square back obstacle is not dominated by longitudinal three-dimensional vortical structures, therefore a preliminary two-dimensional study can be useful to supply information and general trends for a further control study in three dimensions around a hemisphere. The aim is to use a control device easy to set up, low cost and allowing to keep the geometry unchanged. As active control devices can be hardly implemented in such a case, an efficient passive strategy seems to be a good alternative. A suitable solution has already been proposed by Bruneau and Mortazavi in [3-7]. It consists in adding a porous sheath on the obstacle surface in order to reduce the vorticity generation of the boundary layer. The presence of a porous medium at the solid-fluid interface indeed imposes a kind of mixed boundary condition intermediate between the no-slip and the slip one on the solid boundary [8]. As a result, the shear forces are decreased and the flow dynamics is smoothed. Consequently, the problem we have to solve involves three different media, namely the solid obstacle, the porous layer and the fluid. An easy way to tackle it is to use the penalization method [9]. This method is based on a unique model, the Brinkman-Navier-Stokes equations, which are obtained by adding in the Navier-Stokes equations a penalization term, depending on the intrinsic permeability. Three values of this coefficient represent the three different media. This method can be easily implemented since it enables to consider the governing equations in the whole computational domain. Moreover it does not require to prescribe a boundary condition at the solid boundary or a condition at the porous-fluid interface. The penalization method has been recently implemented in vortex methods to deal with fluid-structure interaction problems [10-12]. It was extended to deforming bodies and applied to find optimal swimming modes of fishes $[13,14]$. In the present work, we consider a two-dimensional viscous and incompressible flow past a semi-circular cylinder. We also introduce some porous coatings on the obstacle in order to manipulate the flow. The Brinkman-Vorticity Transport Equations are solved using remeshed vortex methods [15-17] which are characterized by their efficiency and robustness at high Reynolds numbers since they allow to focus the computational task on the rotational zones and to solve the flow equations in a fast Lagrangian way.

In the following we first describe the methodology and show some validations and convergence studies. Then we present the results of two-dimensional passive flow control for several devices differing from each other in terms of the permeability, the thickness and the geometrical configuration of the added porous layer. A careful comparison is carried out between controlled and uncontrolled flows at transitional $(\operatorname{Re}=550)$ and highly transitional regime $(\operatorname{Re}=3000)$.

\section{Vorticity Formulation And Vortex Methods}

The dynamics of an incompressible flow is governed by the incompressible Navier-Stokes equations :

$$
\begin{aligned}
\nabla \cdot \mathbf{u} & =0 \quad \text { in } D \\
\frac{\partial \mathbf{u}}{\partial t}+(\mathbf{u} \cdot \nabla) \mathbf{u} & =-\nabla p+\frac{1}{\operatorname{Re}} \Delta \mathbf{u} \quad \text { in } D,
\end{aligned}
$$

where $D$ is the computational domain, $\mathbf{u}$ the velocity and Re the Reynolds number. In this work, flow simulations are based on particle methods. The fluid particles which are displaced by convection and diffusion are characterized by their position and their vorticity. The vorticity transport is expressed by the Helmholtz equation (or Vorticity Transport Equation), obtained taking the curl of the incompressible Navier-Stokes equations (Eqn. 2) and given in 2D by :

$$
\frac{\partial \omega}{\partial t}+\mathbf{u} . \nabla \omega=\frac{1}{\operatorname{Re}} \Delta \omega \text { in } D
$$

where $\omega$ denotes the vorticity. The Poisson equation

$$
\nabla^{2} \mathbf{u}=-\nabla \times \omega
$$


obtained from continuity equation (Eqn. 1), enables to recover velocity field once the vorticity field is known. The previous equations are approximated using a Vortex method $[15,18]$. These methods are very robust and low-cost to simulate high Reynolds number recirculating flows since the computationel task is mainly focused on vortical structures (see for example $[19,20]$ ). In this kind of approach, the VTE equation (Eqn. 3) is solved using a two-fractional step (or viscous splitting) method. It relies on approximating separately the diffusion and convection terms at each time step. The convective part is solved using a "Vortex-In-Cell (VIC)" method (see e.g. [15] and [21] for its application to active flow control) with a semiLagrangian resolution. In this fractional step a convective velocity is associated to each finite vortex element through a high order interpolation procedure, and the displacement is achieved using a Runge-Kutta method. In order to avoid Lagrangian distortion, particles are then remeshed on the original grid using the same interpolation kernel as the one used previously to interpolate grid velocity values onto the particles. Finally, for computational efficiency and accuracy, diffusion and Poisson equation are solved on the grid using Fast Fourier Transforms (FFT).

\section{Vortex Penalization Method in Vorticity Formulation}

Before all, we show how the penalization method can be used successfully to model the flow of an incompressible fluid around an obstacle [9]. In the penalization technique the system is considered as a single flow, subject to the Navier-Stokes equation with a penalization term that enforces continuity at the solid-fluid interface and rigid motion inside the solid. In this work, the penalization term is expressed using vorticity formulation. The main interest of the penalized vorticity formulation is that it replaces the usual vorticity creation algorithm in order to satisfy the no-slip boundary condition for vortex methods. This new technique avoids the convergence difficulties due to the particle creation on the solid boundaries (see [10] and [17]). We solve simultaneously the Brinkman equations in the solid and the Navier-Stokes equations in the fluid, considering whole the domain as a porous medium with zero (solid) or infinite permeabilities (fluid). Thus, defining the Reynolds number as $\operatorname{Re}=u_{r e f} l_{r e f} / v$, the non-dimensional penalized vorticity equation (or Brinkman-Navier-Stokes equation) reads

$$
\frac{\partial \omega}{\partial t}+(\mathbf{u} . \nabla) \omega=\frac{1}{\operatorname{Re}} \Delta \omega+\nabla \times\left[\lambda \chi_{S}\left(\mathbf{u}_{\mathbf{s}}-\mathbf{u}\right)\right]
$$

where $\chi_{S}$ denotes the characteristic function that yields 0 in the fluid and 1 in the solid, $\mathbf{u}_{\mathbf{s}}$ indicates the rigid body velocity which is zero in all this work since the body is fixed and $\lambda=\mu \Phi l_{r e f} / \rho k u_{r e f}$ is the non-dimensional penalization parameter, in inverse proportion to the permeability of the medium (with $k$ the intrinsic permeability, $\mu$ the viscosity, $\Phi$ the porosity of the porous material, $l_{r e f}$ the height of the obstacle, $\rho$ the density and $u_{r e f}$ the reference velocity). The main advantage of this method is that it needs neither the mesh to fit the boundaries nor to specify no-slip boundary conditions. In addition it allows to compute the pressure as a continuous field on the whole domain including the solids.

The zone variation is realized changing the penalization coefficient that defines the permeability of each region. Numerically, the fluid is considered as a porous medium with a very high permeability $(\lambda=0)$ and the bodies are considered as porous media with a very small permeability $\left(\lambda=10^{8}\right)$.

To discretize the penalized vorticity equation (Eqn. 5) in a vortex method, the equation is split in substeps. At each time step, one successively solves the unsteady penalization-convection-diffusion equations:

$$
\begin{array}{r}
\frac{\partial \omega}{\partial t}=\nabla \times\left(\lambda \chi_{S}\left(\mathbf{u}_{\mathbf{s}}-\mathbf{u}\right)\right) \\
\frac{\partial \omega}{\partial t}+(\mathbf{u} \cdot \nabla) \omega=0 \\
\frac{\partial \omega}{\partial t}=\frac{1}{R e} \Delta \omega .
\end{array}
$$

To solve Eq. 6 we use an implicit scheme ( [10]) and we set:

$$
\widetilde{\omega}^{n+1}=\nabla \times\left[\frac{\mathbf{u}^{n}+\lambda \Delta t \chi_{S} \mathbf{u}_{\mathbf{s}}{ }^{n}}{1+\lambda \Delta t \chi_{S}}\right]
$$

where $\Delta t$ is the time step. The right hand side above is evaluated by second order centered finite differences.

At this stage, grid vorticity above a certain cut-off is used to create particles in grid point surrounding cells and Eq. 7 is solved by a classical vortex-in-cell method [15]. Particles are pushed with a RK4 time-stepping and are then remeshed on the original grid using the third order interpolation kernel $M_{4}^{\prime}[22]$. Then, diffusion (Eq. 8) and Poisson equation (Eq. 


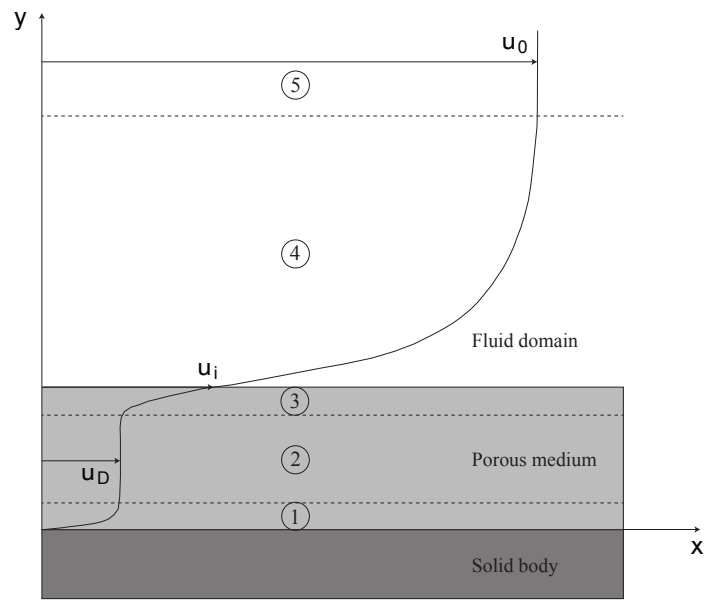

Fig. 1. Velocity profile in the vicinity of a porous medium.

4) are solved on the grid using FFT-based evaluations as described in [23]. Grid values for vorticity and velocity are now available for time $t_{n+1}$ and a new cycle of iterations can start. Moreover, the no-slip boundary conditions are naturally satisfied penalizing the vorticity transport equations.

This hybrid vortex penalization method has already been successfully used to simulate high Reynolds flow regimes past bluff bodies [12,24].

\section{Vortex Penalization Method for Solid-Porous-Fluid Media}

This section is devoted to the study of flows in solid-porous-fluid media and aims to highlight the efficiency of the vortex penalization method to solve such problems. For three different solid, porous and fluid media configurations, it is of great importance to clearly understand the flow behavior at the fluid-porous interface in order to model the physics correctly. As described in [6], we can consider five different flow regions from the solid to the free flow in the fluid. The first one is the boundary layer inside the porous medium, close to the solid wall. This one is very thin compared to the second region which is characterized by the homogeneous porous flow with Darcy velocity (numbers 1 and 2 in Fig. 1). In the vicinity of the porous-fluid interface, two transient layers can be recognized (numbers 3 and 4 in Fig. 1). The first one corresponds to the increase of the porous layer velocity reaching $\mathbf{u}_{i}$ value at the interface and the second one to the fluid boundary layer standing from the interface to the free flow. The fluid boundary layer growth is then determined by $\mathbf{u}_{0}-\mathbf{u}_{i}$ where $\mathbf{u}_{0}$ denotes the velocity of the main fluid flow (number 5 in Fig. 1). The aim is thus to find out a way to solve the flow both in the porous medium, the fluid region and at the interface between the two media. Several approaches have already been proposed to handle this problem like avoiding to solve the porous flow enforcing appropriate porous-fluid boundary conditions [25] or solving the governing equations of each region coupling Darcy equations and Navier-Stokes equations with a right treatment at the interface $[26,27]$. Nevertheless, these two approaches present some drawbacks since the first one neglects the porous medium physics and does not permit to have overall view of the problem and the second one is particularly difficult to handle especially because of the interface problem. The method presented here, based on the vortex-penalization technique, appears as a suitable alternative since it involves a unique equation (Eq. 5) for the whole domain and ensures an accurate modeling of each of the different regions thanks to the dimensionless penalization factor $\lambda$. Here, $\lambda$ is expressed as $\lambda=\mu \Phi l_{\text {ref }} / \rho k u_{\text {ref }}$ where $l_{r e f}, \rho, u_{r e f}=1$ in this study and the porosity $\Phi$ is close to 1 as imposed by Brinkman equations [28]. Therefore $\lambda$ essentially depends, in the inverse proportion, on the intrinsic permeability $k$ of the medium. Varying the $\lambda$ value directly defines the different media according to the following equation obtained using implicit Euler scheme for the penalization velocity discretization:

$$
\widetilde{\mathbf{u}}^{n+1}=\frac{\mathbf{u}^{n}}{1+\lambda \Delta t \chi_{S}} .
$$

Indeed, in the fluid, the intrinsic permeability coefficient $k$ goes to infinity, thus the fluid can be considered numerically as a porous media with a very high permeability. We set $\lambda=0$ in this region. According to Eq. 10, the velocity in the fluid is not penalized $\left(\widetilde{\mathbf{u}}^{n+1}=\mathbf{u}^{n}\right)$ and since $\lambda=0$, the penalization term vanishes in Eq. 5, and we naturally recover the Vorticity Transport Equation (Eq. 3). On the contrary, the solid has a permeability coefficient $k$ which goes to zero, it can be consequently modeled setting the penalization parameter $\lambda$ to a very high value. In this study $\lambda$ equals $10^{8}$ in the solid, which vanishes the flow velocity in this region according to Eq. $10\left(\widetilde{\mathbf{u}}^{n+1} \rightarrow 0\right)$. 
It has been proved in [9] that solving Eq. 5 with such a value of $\lambda$ was equivalent to solve Darcy's law in the solid. Furthermore, setting the $\lambda$ parameter to an intermediate value, reasonably chosen between these two extreme values $(\lambda=0$ and $\lambda=10^{8}$ ), would model a porous medium in which the flow has a Darcy velocity $\mathbf{u}_{D}$ (Fig. 1). For example $\lambda=1000$ corresponds to a very low permeable medium and $\lambda=1$ represents a very high permeability. The variation of $\lambda$ corresponds to the variation of $k$ that specifies the intrinsic porous material permeability. The accuracy and efficiency of the penalization method come from its capability to take into account these variations of $\lambda$ and to capture the induced steep velocity variations at the different interfaces with a minimum number of discretization points.

\section{Validation and grid convergence study}

In order to verify the accuracy of the method, we present in this section a validation study of the computational method employed in this work. First we consider flows past semi-circular and circular cylinders at different regimes, and we compare the results with the ones of the literature. Secondly we present a grid convergence study for flow past a semi-circular cylinder, the obstacle of interest in this work. Each simulation presented in the following is performed considering the same geometrical setup: the obstacle under study has a dimensionless diameter of $d=1$ and its center (in the case of a circular cylinder) or its back wall (in the case of a semi-circular cylinder) is located at $(x, y)=(0,0)$ in the computational domain $D$. The whole domain is meshed by an equispaced Cartesian orthogonal grid. As we use FFT-based evaluations to solve diffusion and Poisson equations, periodic boundary conditions are considered on the box walls and a correction of velocity is performed at each time step in order to satisfy the free stream velocity $\mathbf{u}_{\infty}=\left(u_{r e f}, 0\right)=(1,0)$ imposed at the inlet.

\subsection{Validation for flow past a circular and a semi-circular cylinder}

The first part of this validation study deals with incompressible flow past a semi-circular cylinder.We compare our results to numerical and experimental results of Farhadi et al. [29] and Boisaubert and Texier [30]. The flow analysis presented hereafter are based on the near-wake length measurement, the Strouhal number $S_{t}$ (given by the shedding frequency) and the drag and lift coefficients, expressed by:

$$
C_{D}=\frac{2 F_{D}}{\rho u_{r e f}^{2} d}, \quad C_{L}=\frac{2 F_{L}}{\rho u_{r e f}^{2} d} .
$$

where the drag and lift forces, respectively noted $F_{D}$ and $F_{L}$, are computed according to the momentum equation given by Noca et al. in [31]. The Reynolds number ranges between 100 and 300 in this study.

As can be seen in Table 1, the results obtained at $\mathrm{Re}=100$ and $\mathrm{Re}=300$ show a good agreement with the numerical values given by [29]. A more detailed comparison is performed at $\mathrm{Re}=200$ and the related results are reported in Fig. 2. Concerning in particular drag and lift coefficients, one can see from the left picture in this figure that our results coincide very well with those of [29]. Indeed we find $\overline{c_{D}}=1.85 \pm 0.15, \overline{c_{L}}=0 \pm 0.53$ and $S_{t}=0.230$ to be compared with $\overline{c_{D}}=1.85 \pm 0.12, \overline{c_{L}}=0 \pm 0.50$ and $S_{t}=0.215$ in [29]. The right hand side picture of Fig. 2 gives a comparison of the normalized near-wake length $L$ with the numerical results of [29] and the experimental data of [30]. The near-wake length is defined as the $x$-coordinate of the point located on the centerline in the $x$-direction where the streamwise component of the velocity vanishes. Again, our results agree rather well with the literature.

We complete this validation study by investigating the circular cylinder benchmark for which references addressing higher Reynolds numbers can be found in the literature. The Reynolds numbers considered in this part are the one we selected to perform the passive control study, that is to say $\operatorname{Re}=550$ (transitional regime) and $\mathrm{Re}=3000$ (highly transitional regime). The early-time evolution of the drag coefficient for both regime is reported in Fig. 3 and one can see that the results are in excellent agreement with the considered references.

\begin{tabular}{lllll}
\hline & \multicolumn{2}{r}{$\mathrm{Re}=100$} & \multicolumn{2}{c}{$\mathrm{Re}=300$} \\
\hline Authors & - & $S_{t}$ & $\overline{c_{D}}$ & $S_{t}$ \\
\hline Farhadi et al. [29] & 1.73 & 0.180 & 1.955 & 0.22 \\
Present method & 1.70 & 0.192 & 1.90 & 0.24 \\
\hline
\end{tabular}

Table 1. Comparison of mean drag coefficient $\left(\overline{c_{D}}\right)$ and Strouhal number $\left(S_{t}\right)$ for flow past a semi-circular cylinder at $\mathrm{Re}=100$ and $\mathrm{Re}=300$. 

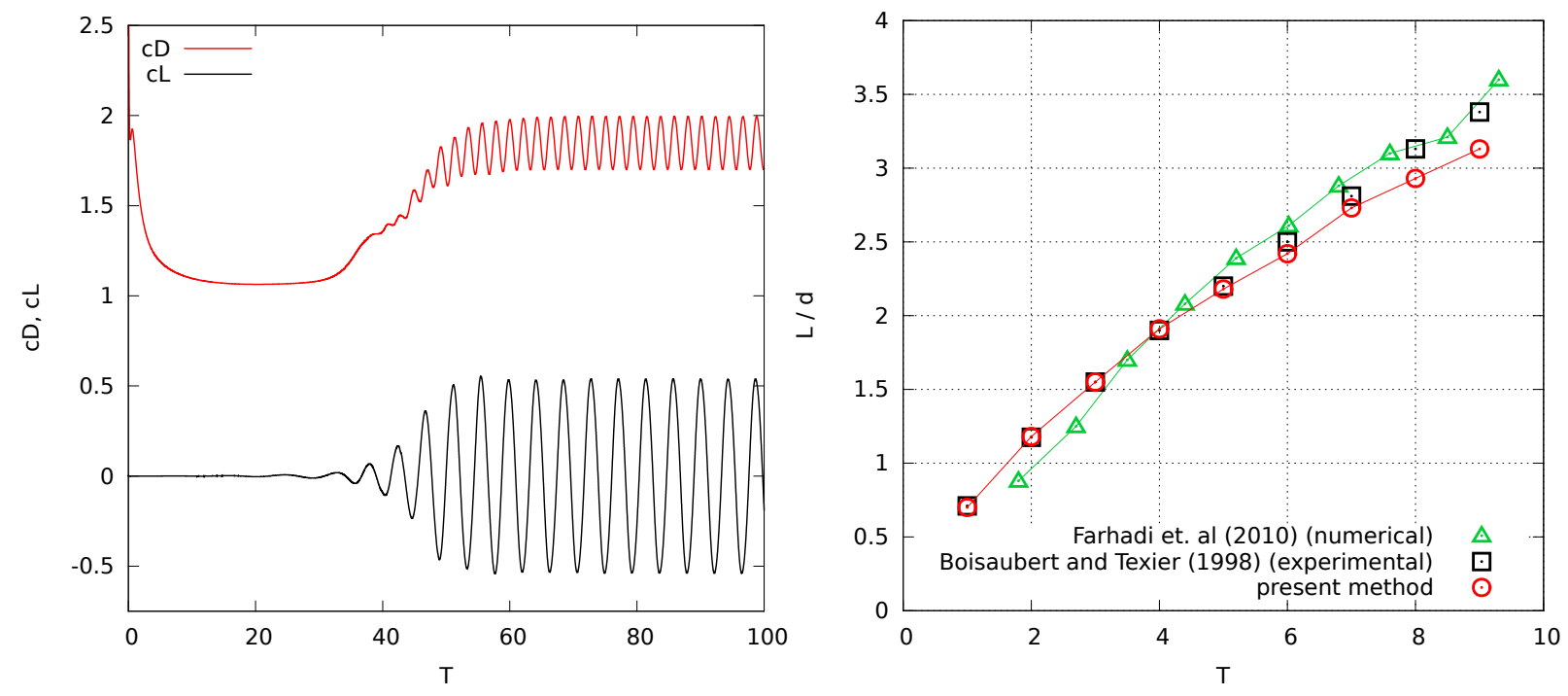

Fig. 2. (Left) Drag and lift evolution for flow past a semi-circular cylinder at $R e=200$. (Right) Normalized near-wake length $L / d$ of a semicircular cylinder at $\mathrm{Re}=200$ : comparison with numerical results [29] (green triangles) and experimental data [30] (black squares).

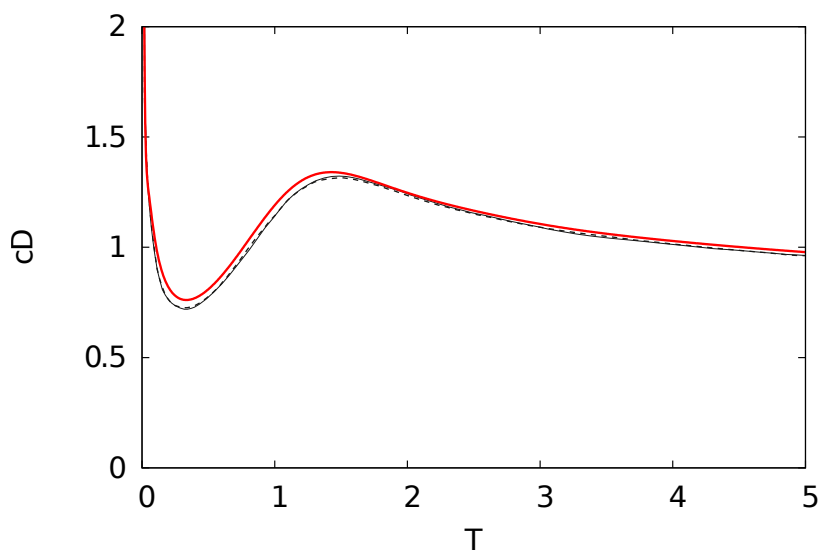

(a) $\operatorname{Re}=550$

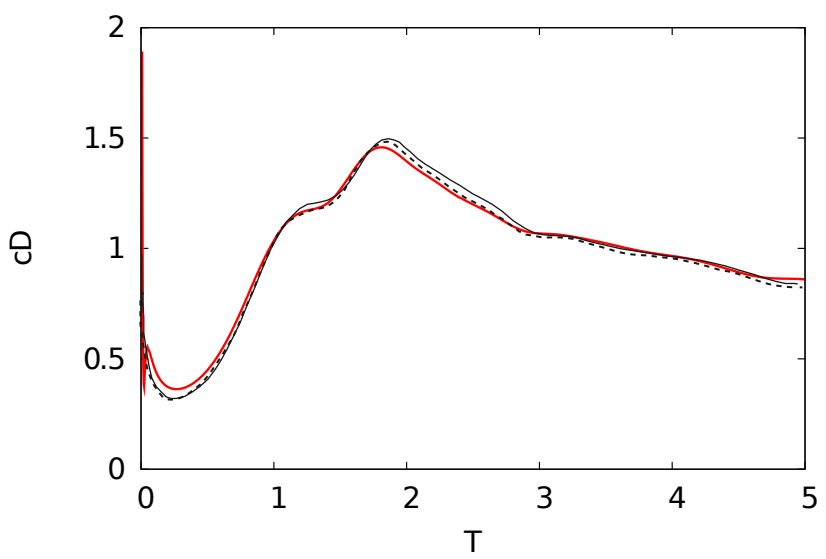

(b) $\operatorname{Re}=3000$

Fig. 3. Drag evolution for flow past a circular cylinder at $R e=550$ and $R e=3000$. Comparisons of present method (solid red line) with Koumoutsakos \& Leonard [16] (dashed line) and Ploumhans \& Winckelmans [32] (solid black line).

\subsection{Grid convergence}

We perform herein a grid convergence study of the vortex penalization method for flow past a solid semi-circular cylinder at $\operatorname{Re}=550$ and $\operatorname{Re}=3000$. This grid convergence is carried out on three grid levels. For $\mathrm{Re}=550$ the three consecutive mesh sizes are: $h=0.01, h=0.005$ and $h=0.0025$ in the computational domain $D=[-4,8] \times[-5,5]$. For $\operatorname{Re}=3000$, as the boundary layer is thinner, we perform the convergence study with three finer mesh sizes, respectively $h=0.005$, $h=0.0025$ and $h=0.00125$. The simulations are carried out in a smaller domain $D=[-2.5,5] \times[-3,3]$ in order to reduce the computational cost induced by the finest grid. The results reported in Table 2, give the mean values of drag and enstrophy. For $\operatorname{Re}=550$, the results on the two finest consecutive grids are very close (Table 2). We can consider that the grid convergence is achieved and the adopted grid size for the further flow control simulations is $h=0.005$ with $\Delta t=0.0025$. For $\operatorname{Re}=3000$ the grid convergence is achieved with $h=0.0025$ and $h=0.00125$. The corresponding studies will thus be performed using $h=0.0025$ as the grid size and $\Delta t=0.002$ as the time step. Let us emphasize the interest of the vortex methods which enables here to handle a highly transitional regime using a relatively large time step compared to classical Eulerian methods. 


\begin{tabular}{lllll}
\hline & \multicolumn{3}{c}{$\mathrm{Re}=550$} & \multicolumn{2}{c}{$\mathrm{Re}=3000$} \\
\hline Grid & $\overline{c_{D}}$ & $\bar{Z}$ & $\overline{c_{D}}$ & $\bar{Z}$ \\
\hline$h=0.01$ & 1.49 & 122 & - & - \\
$h=0.005$ & 1.91 & 158 & 1.89 & 313 \\
$h=0.0025$ & 1.98 & 161 & 1.94 & 292 \\
$h=0.00125$ & - & - & 1.96 & 280 \\
\hline
\end{tabular}

Table 2. Grid convergence study for flow past a semi-circular cylinder at $R e=550$ and $R e=3000$ through the mean values of drag coefficient $\left(\overline{c_{D}}\right)$ and enstrophy $(\bar{Z})$.

\section{Passive control}

In this work, the vortex penalization method is applied to passive flow control around a two-dimensional semi-circular cylinder. The control is achieved covering this obstacle with a porous coating. The expected effect of a porous medium at the solid-fluid interface is to modify the vorticity generation of the boundary layer and the vortex shedding, and therefore to reduce drag forces and vortex induced vibrations improving the aerodynamic properties of the obstacle.

The subsequent flow control simulations are performed at transitional $(\mathrm{Re}=550)$ and highly transitional regime $(\operatorname{Re}=$ 3000). As at these two regimes the flow is not turbulent, Direct Numerical Simulations (DNS) are performed to numerically solve Eq. 5. The semi-circular cylinder has a total dimensionless diameter of $d=1$ including a porous layer of thickness $\tau$, whose back wall is centered at $(x, y)=(0,0)$ in the computational domain $D=[-4,8] \times[-5,5]$ depicted in Fig. 4 .

In order to analyze the effects of the control approach we will compare velocity, vorticity and pressure fields as well as global flow quantities like the drag force $\left(F_{x}\right)$ and the enstrophy $(Z)$ which measures the dissipation effects in the flow and defined by $Z=\int_{D}|\omega|^{2} \mathrm{~d} \mathbf{x}$. Note that in this study we will consider the drag force instead of the drag coefficient since the computation formula of the latter involves the diameter $d$ of the obstacle which includes the porous layer.

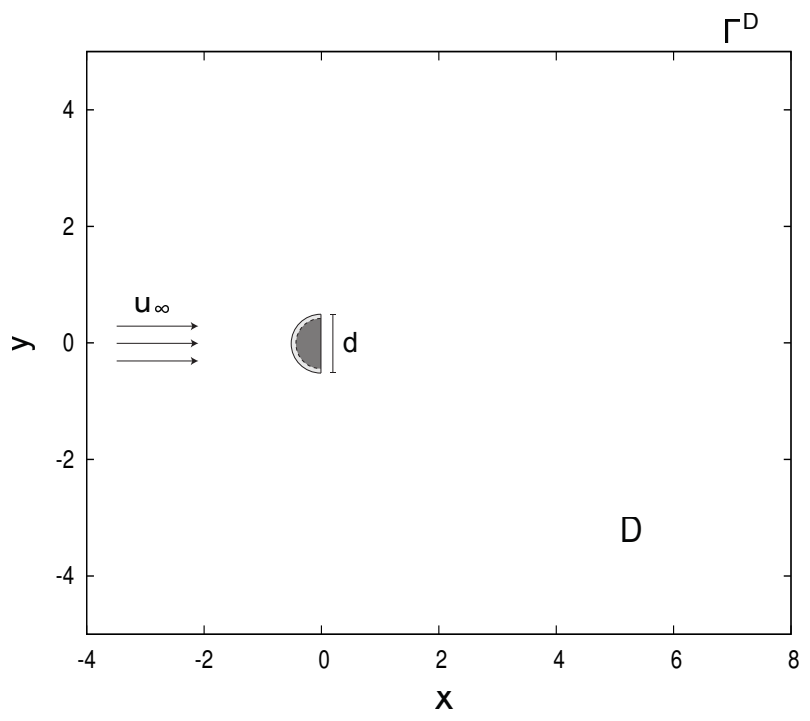

Fig. 4. Computational domain used for passive flow control.

\subsection{Parametric study on penalization parameter $\lambda$ for homogeneous porous layer}

This section relies on the influence of the added porous layer permeability on the flow control behaviour and the efficiency of such a passive control. We perform a parametric study at $\mathrm{Re}=550$ and $\mathrm{Re}=3000$ considering four consecutive values of $\lambda$ inside the porous coating, namely $\lambda=1$ (high permeability), 10,10 $0^{2}, 10^{3}$ (low permeability) and compare the results to the fluid case $(\lambda=0)$ and the uncontrolled case. The fluid case means a semi-cylinder including a porous layer with permeability $\lambda=0$, which corresponds to a smaller semi-cylinder with diameter $d-2 \tau$. In this section, the thickness of the layer is set to $\tau=10 \% d=0.1$ for each case (Fig. 5).

As can be seen in Fig. 6, which represents dimensionless time history of global flow quantities at $\mathrm{Re}=550$, setting $\lambda=1$ inside the layer clearly appears as the best solution in terms of flow regularization. Indeed, the mean value of drag 


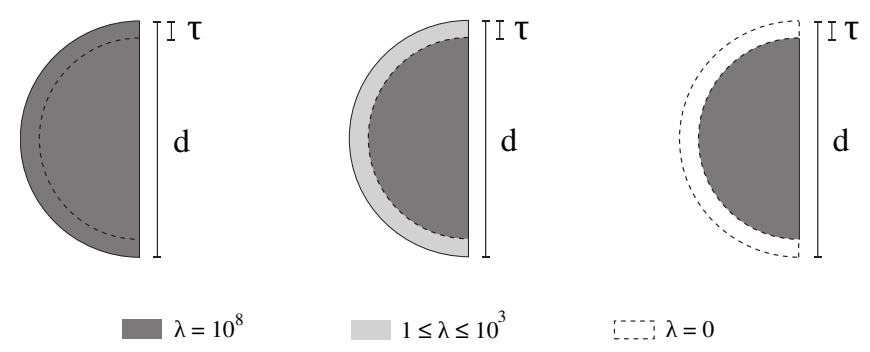

Fig. 5. (left) Uncontrolled case, (center) porous case, (right) fluid case.
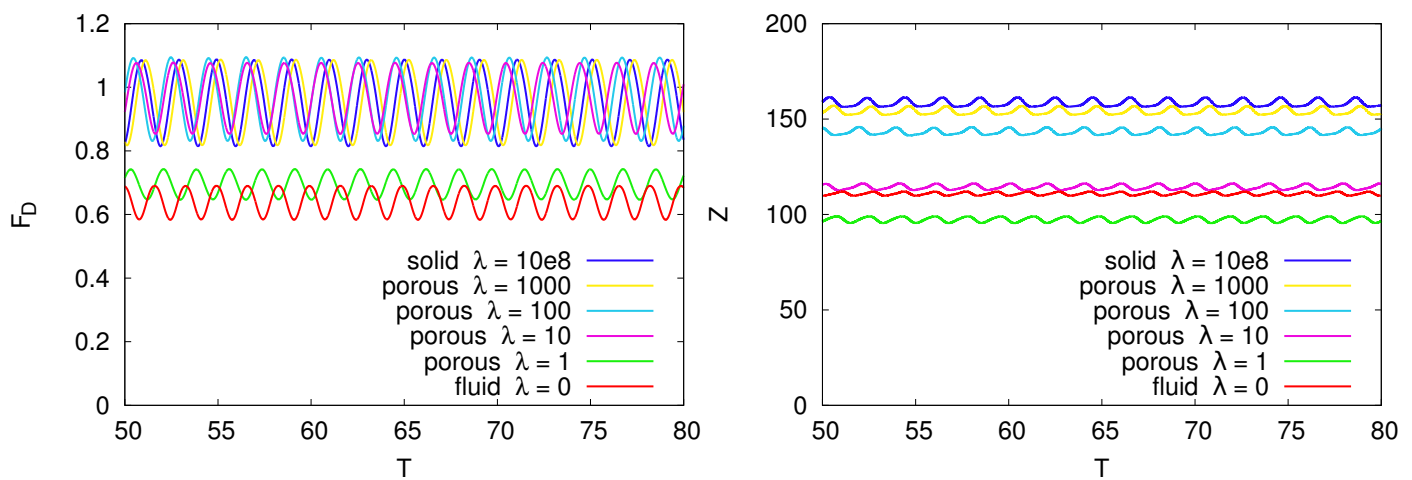

Fig. 6. Effects of various layer permeabilities on drag force (left) and enstrophy (right) for the flow past a semi-circular cylinder at Re=550.
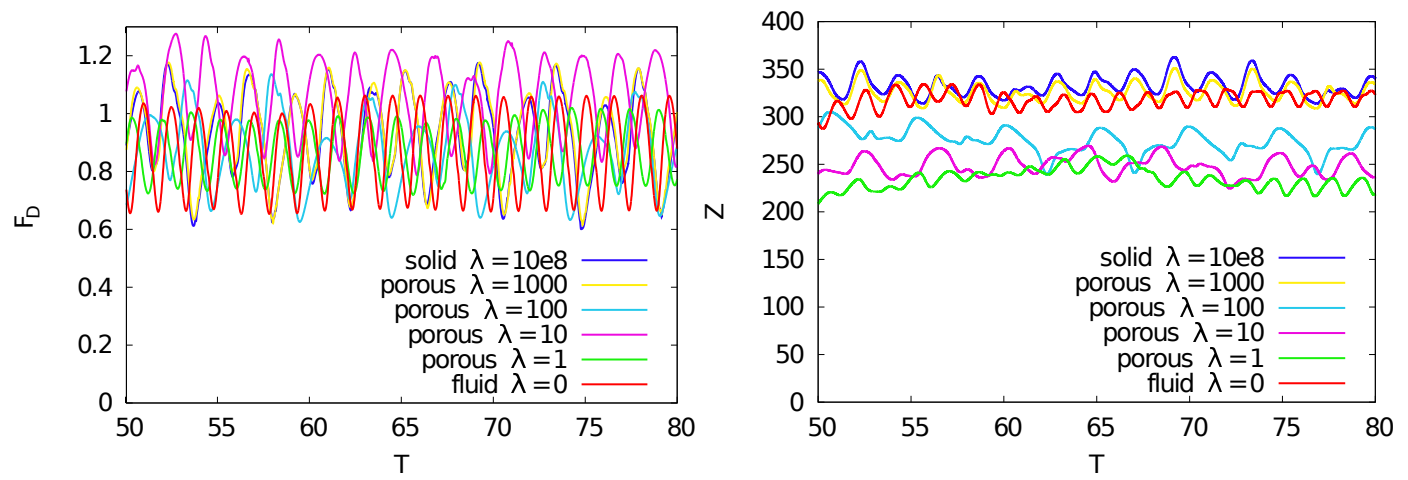

Fig. 7. Effects of various layer permeabilities on drag force (left) and enstrophy (right) for the flow past a semi-circular cylinder at Re=3000.

force (Fig. 6 (left)) reaches for $\lambda=1$ an optimum value close to the one of the fluid case, showing a drag reduction of $27 \%$ compared to uncontrolled case. For all the other values of porous $\lambda$, the drag reduction effects are nearly nonexistent. Results of enstrophy evolution (Fig. 6 (right)) show a progressive reduction of the dissipation and the delay to transition with the decrease of $\lambda$ values. Moreover, we note that the result with $\lambda=1$ is even better than the one of fluid case and represents an improvement of nearly $40 \%$ compared to uncontrolled case.

At $\operatorname{Re}=3000$ the best solution for global flow regularization is also achieved setting $\lambda=1$ inside the porous coating. This configuration leads to a drag and enstrophy reduction of respectively $6 \%$ and $30 \%$ compared to the uncontrolled case and one can see on Fig. 7 the important decrease of the signals amplitude. In terms of control effects, the main difference one can notice here in comparison to the parameter study performed at $\operatorname{Re}=550$ concerns the flow behaviour observed when $\lambda=10$ inside the layer. Contrary to the other $\lambda$ values, the flow field obtained with the latter is non-periodic (Fig. 7). This flow irregularity impacts the mean drag value, which is increased of about $15 \%$ compared to the uncontrolled case. We can summarize that except for a very permeable porous layer $(\lambda=1)$, a porous coating covering the whole face of the semi-cylinder is not a convenient tool to reduce the drag. It is only useful to regularize the wake. The problem seems due to the separation point that is located inside the porous medium. 


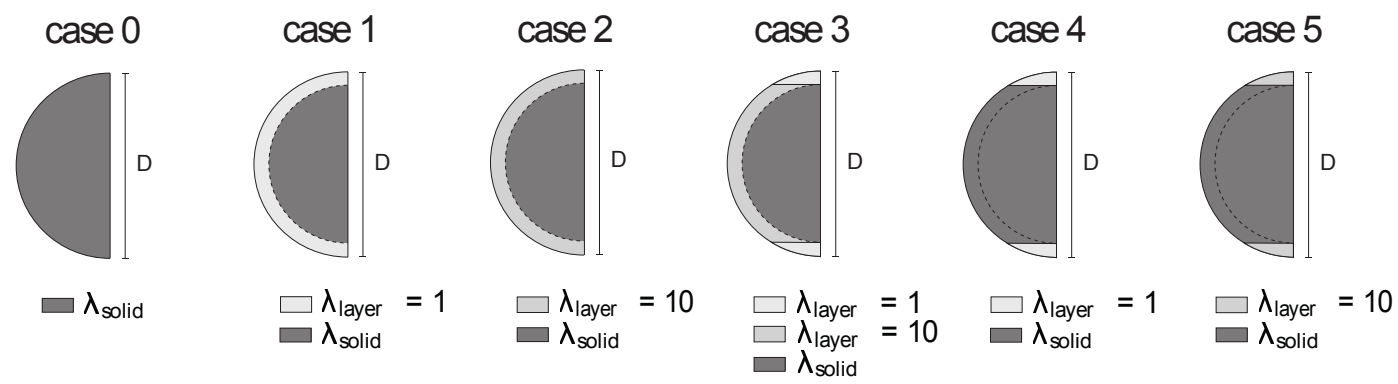

Fig. 8. Cases 1 to 5 corresponding to different porous layer configurations. Case 0 depicts the uncontrolled case.

\subsection{Influence of the porous layer configuration on flow control efficiency}

This section aims to determine control devices which are both efficient in terms of flow regularization and easy to set up in terms of manufacturing constraints. According to the parametric study presented in the previous section, setting the $\lambda$ function to 1 in the surrounding sheath enables important flow reduction effects. Nevertheless, this configuration turns out to be industrially difficult to implement because of the very high permeability of the added layer. On the contrary, intermediate permeabilities obtained fixing $\lambda=10$ inside the porous interface are easier to handle but show quite poor improvements in terms of flow control, as reported in the parametric study. Taking into account these observations, we propose to compare the six test cases depicted in Fig. 8. Case 0 refers to the uncontrolled case, cases 1 and 2 correspond to homogeneous layer cases where $\lambda$ respectively equals 1 and 10 , and cases 3 to 5 refer to heterogeneous cases where the porous layer is split in two regions with different permeabilities. The simulations were performed taking the same numerical parameters as the one used for the parametric study. In a first place, the thickness of the layer is kept to $10 \% d=0.1$. The asymptotic mean values of global flow quantities and the time averaged vorticity, velocity and pressure fields are respectively given for each case in Table 3, and Figures 9 to 14.

First of all, the results reported here give further information concerning cases 1 and 2 . They confirm that case 2 is not competitive as it increases the drag for $\mathrm{Re}=550$ and $\mathrm{Re}=3000$ compared to uncontrolled case (Table 3 ). They also highlight the efficiency of case 1 which induces a significant reduction of drag force $(-27 \%$ and $-6 \%)$ and enstrophy $(-38 \%$ and $-29 \%$ ). These quantitative results are confirmed by the mean vorticity and velocity fields showing the smoothing of wake dynamics generated by the presence of the highly permeable layer. Indeed, the near wake structures are smaller and the back recirculation zone is sharply reduced (Figs. 9 and 12), implying an increase of downstream pressure (Figs. 11 and 14 ) and thus a reduction of drag forces. Finally, the vortices swirl with lower velocity and for $R e=3000$ the mean wake becomes axisymmetric (Fig 10 and 13).

Let us now focus on the heterogeneous devices. For both regime, the case 3 shows benefits which are very comparable to those of case 1 . We note that the only difference with case 2 is the presence of highly permeable poles in the layer. This change allows an eddy detachment from the wall with larger shedding structures, diminishing the back wall recirculation zone (Figs. 9 and 12) and a drastic increase of downstream near wall pressure for the $\mathrm{Re}=550$ regime (Fig. 11). The case 3 also enables to significantly regularize the flow in the wake with lower velocities, using highly permeable material introduced in both edges of the body (Figs. 10 and 13). Nevertheless, the mix of high and intermediate permeabilities induces difficulties to industrially build such a coating and has no practical interest.

The cases 4 and 5 are more interesting for industrial applications. For these cases, the front part of the coating is no more permeable, but completely solid and porous layers are only placed in the two edge parts of the obstacle, which makes the practical implementation much more easier. Here, for both Reynolds numbers 550 and 3000, the enstrophy and the drag force undergo a tremendous decrease except for the case 5 at $\mathrm{Re}=3000$ where the drag force drop is limited to $7 \%$ because of the lower permeability of the porous layer (Table 3). Figures 9 to 14 confirm these results. They show a significant reduction of the mean velocity of the flow in the near and far wake (Figs. 10 and 13) as well as the decrease of the crosswise dimension of the two counter-rotating vortical structures at the back of the obstacle (Figs. 9 and 12). Also, the near wall pressure values (Figs. 11 and 14), especially for the case 4, are sensibly diminished which is in agreement with drag results. These results emphasize the importance of the positions of the permeable zones that should be chosen considering the flow separation phenomenon as it preferably should not occur inside the porous material. Finally, test case 5 , made of intermediate permeabilities on both edges of the body, corresponds to a more realistic device compared to the case 4 with very permeable edges. It can be considered as a suitable and affordable device for flow control.

\subsection{Influence of the porous layer thickness $\tau$ on flow control efficiency}

In the previous sections, the control effects were studied for different configurations using a constant porous layer thickness $\tau=10 \% d$. In this section, the effect of the thickness for different configurations will be investigated, and a special 


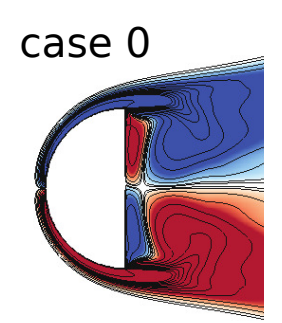

case 1

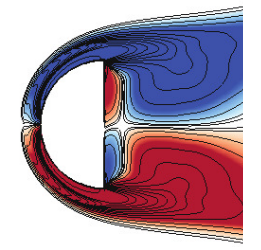

case 2

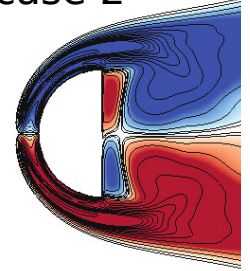

case 3

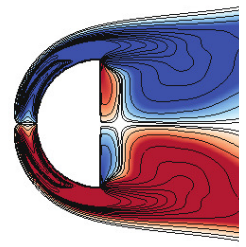

case 4

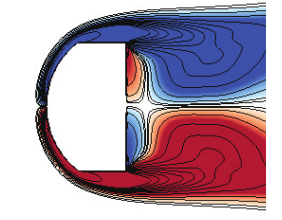

case 5

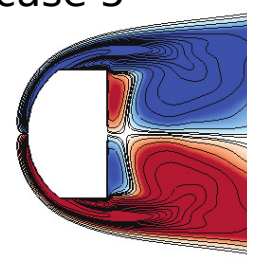

$\omega$

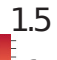

1

0

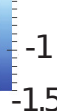

Fig. 9. Zoom of the mean vorticity fields and isolines for the flow past a semi-circular cylinder at $\mathrm{Re}=550$ with $\tau=10 \% d$.
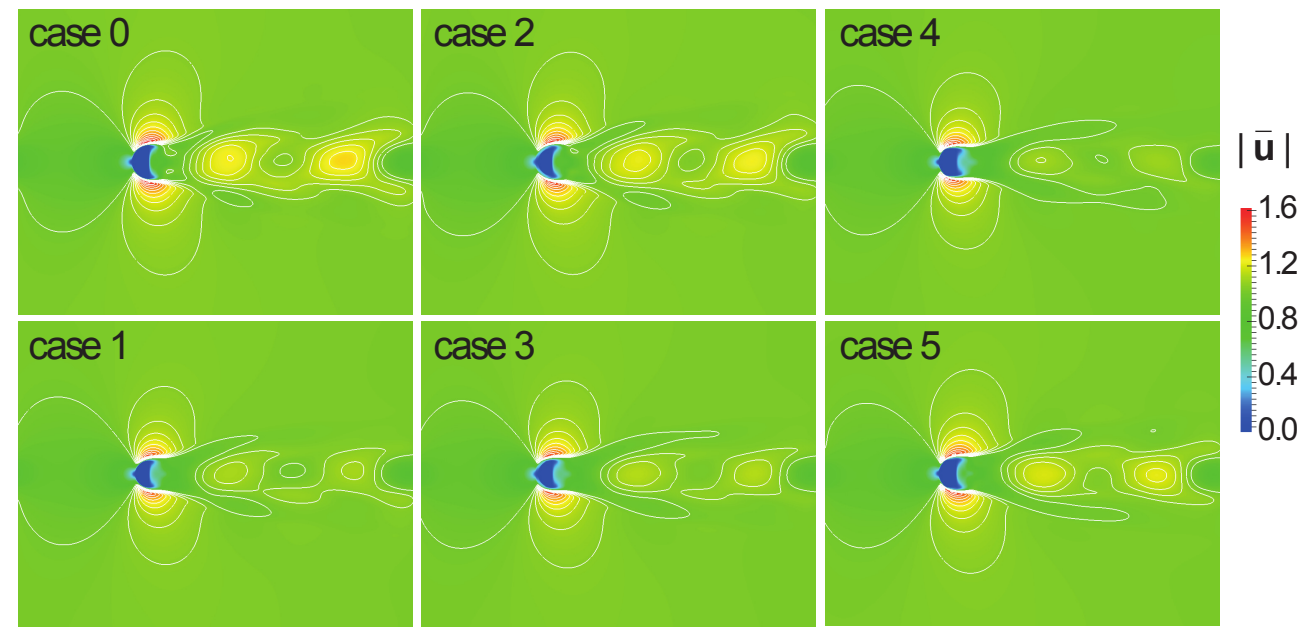

Fig. 10. Fields and isolines of mean velocity magnitude for the flow past a semi-circular cylinder at Re=550 with $\tau=10 \% d$.
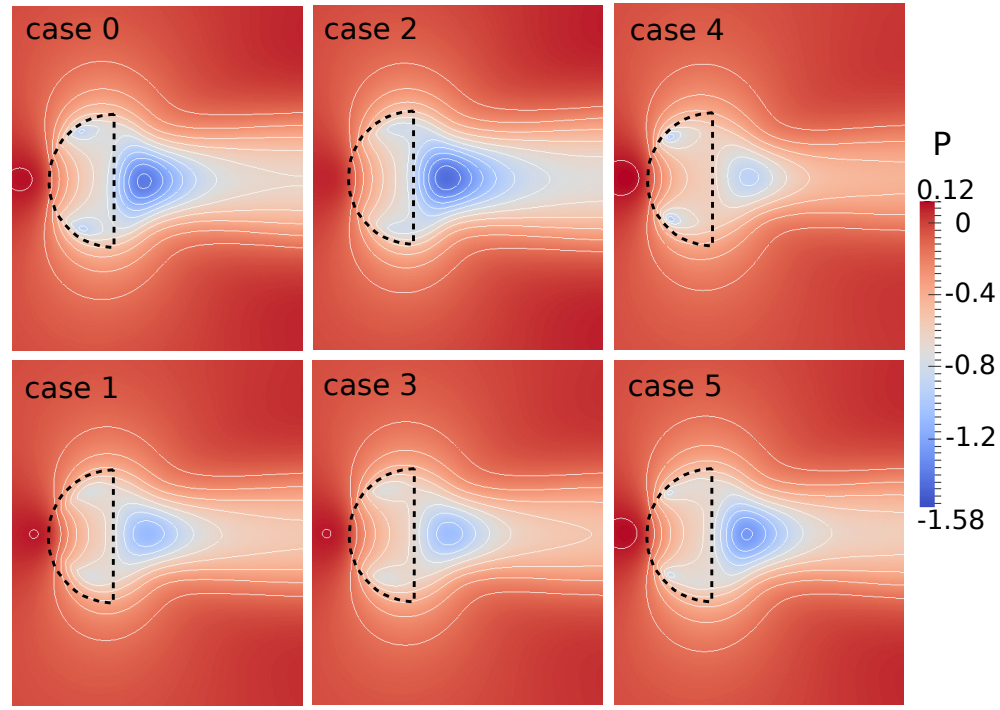

Fig. 11. Zoom of the mean pressure fields and isolines for the flow past a semi-circular cylinder at $R e=550$ with $\tau=10 \% d$. 


\begin{tabular}{lllll}
\hline & \multicolumn{2}{c}{$\mathrm{Re}=550$} & \multicolumn{2}{c}{$\mathrm{Re}=3000$} \\
\hline$\tau=10 \% d$ & $F_{x}$ & Enstrophy & $F_{x}$ & Enstrophy \\
\hline case 0 (uncontrolled case) & 0.957 & 158.4 & 0.926 & 334.2 \\
case 1 & $0.695(-27 \%)$ & $97.5(-38 \%)$ & $0.870(-6 \%)$ & $236.7(-29 \%)$ \\
case 2 & $0.970(+1.4 \%)$ & $114.4(-28 \%)$ & $1.054(+14 \%)$ & $248.8(-26 \%)$ \\
case 3 & $0.668(-30 \%)$ & $87.4(-45 \%)$ & $0.922(-0.4 \%)$ & $223.2(-33 \%)$ \\
case 4 & $0.551(-40 \%)$ & $110.2(-30 \%)$ & $0.737(-20 \%)$ & $270.6(-19 \%)$ \\
case 5 & $0.738(-23 \%)$ & $125.2(-21 \%)$ & $0.860(-7 \%)$ & $281.3(-16 \%)$ \\
\hline
\end{tabular}

Table 3. Reduction effects brought by the different porous layer configurations in comparison to the uncontrolled case at $\mathrm{Re}=550$ and $\mathrm{Re}=3000$ with $\tau=10 \% d$.
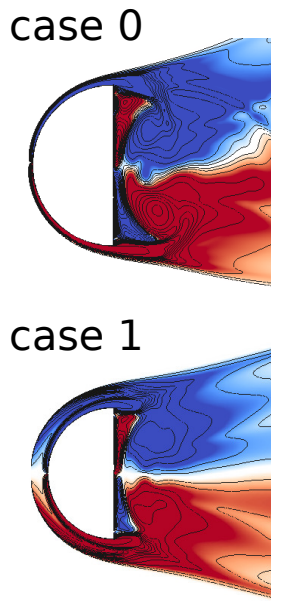

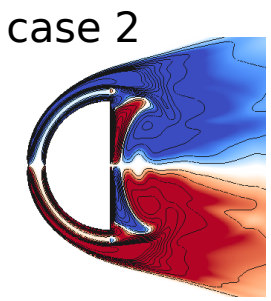

case 3

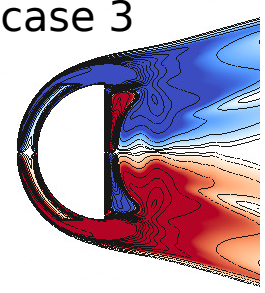

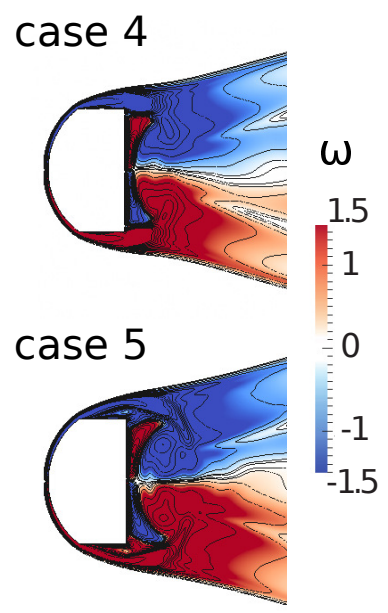

Fig. 12. Zoom of the mean vorticity fields and isolines for the flow past a semi-circular cylinder at $\operatorname{Re}=3000$ with $\tau=10 \% d$.

focus will be made for thin layers in order to reduce the use of porous materials and to find out a compromise between practical constraints and control efficiency. The same test cases (Fig. 5) are now considered setting the thickness of the layer to $\tau=5 \% d=0.05$ and to $\tau=2.5 \% d=0.025$. Table 4 and 5 respectively report the control performances in terms of mean global flow quantities, obtained for each case with $\tau=5 \% d$ and $\tau=2.5 \% d$.

For both regimes $\operatorname{Re}=550$ and $\operatorname{Re}=3000$, when comparing Tables 3, 4 and 5, a slight decrease of control effects is observed. This can be explained looking at the mean profiles of the streamwise component of the velocity, $u$ at $x=-0.05$ plotted in Figures 16 and 18. These profiles show higher acceleration of the flow field inside the layer when its thickness drops, inducing higher velocity gradients in the vicinity of the solid-porous-fluid interface and make the vortex shedding occurring earlier, resulting in a little reduction of beneficial effects. Nevertheless, the decrease of drag and enstrophy remains important for thinner porous layers, which enables to consider them as interesting passive control devices. Also, as shown in Figure 15, a direct correlation between the drag reduction and the back wall pressure forces is noticeable at $\operatorname{Re}=550$ : when drag drops the back wall pressure rises. This fact is more unstable for the $\mathrm{Re}=3000$ as the flow is highly transitional (figure 17). Furthermore, for the later flow regime, decreasing the layer thickness in cases 1, 2 and 3 deteriorate the drag performances as the flow velocity slows down inside the thin layer around the body and the skin friction effects increase the drag forces.

Now let focus on cases 4 and 5. One can observe in Tables 3, 4, 5 that case 4 remains the best control device in terms of drag reduction, regardless of the coating thickness and Reynolds number. As discussed in the previous section, this is due to the fact that the front part of the obstacle is completely solid and that highly permeable zones are precisely located in the vicinity of the boundary layer separation point where the shedding starts. On one hand this configuration enables to efficiently decrease the shedding velocity of the flow damped by porous edges (see Figures 16 and 18), on the other hand it prevents from the negative effect of porous coatings implemented in the front part of the semi-cylinder. For both cases 4 and 5, Figs. 16 and 18 show for all thicknesses a uniform and relatively low acceleration of the flow field inside the layer. As the case 5 is the most practical benchmark (more than all other cases as the porous materials with moderate permeability and edge locations are easier to handle for the manufacturing of a real device), its control efficiency is important to be studied 

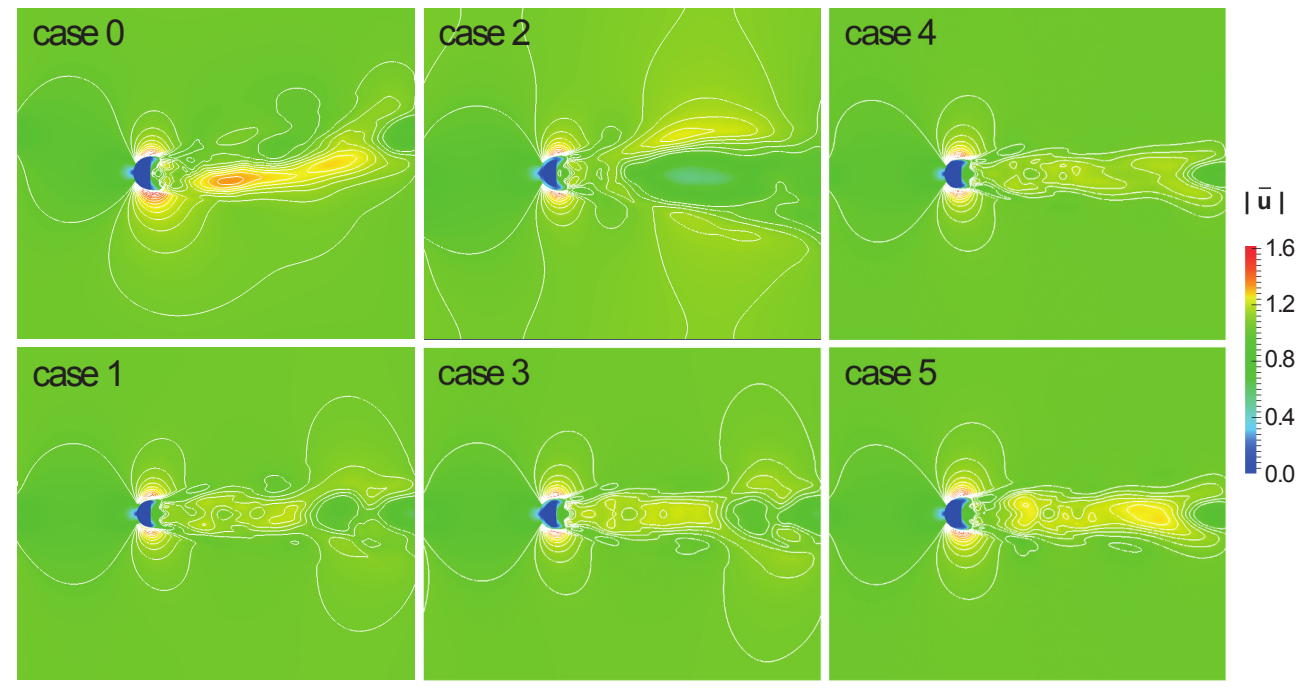

Fig. 13. Fields and isolines of mean velocity magnitude for the flow past a semi-circular cylinder at $\operatorname{Re}=3000$ with $\tau=10 \% d$.
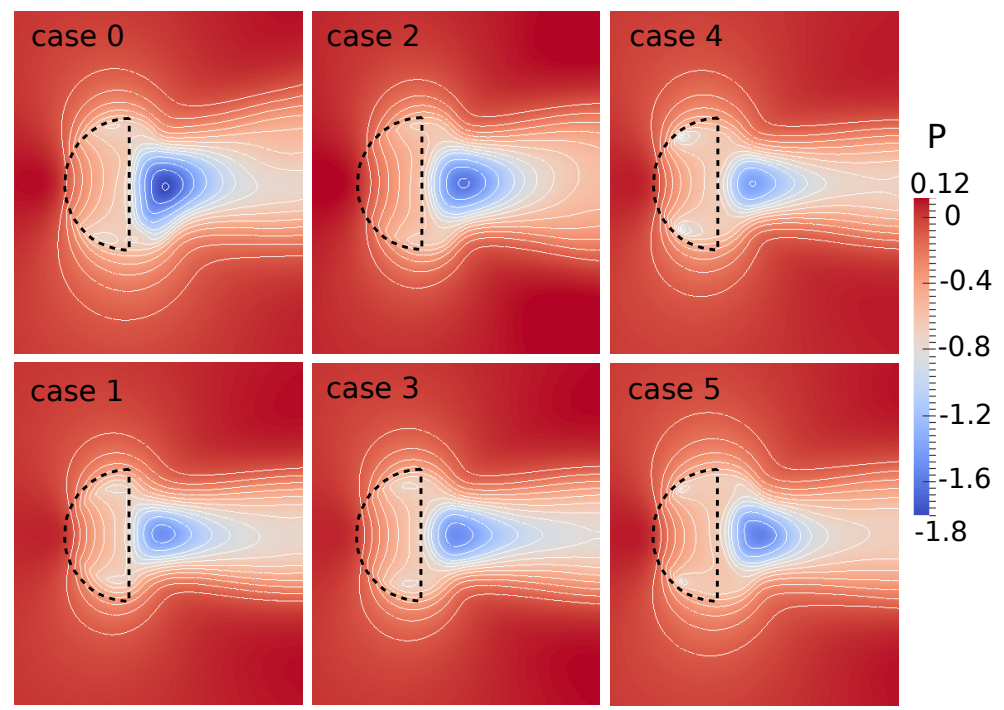

Fig. 14. Zoom of the mean pressure fields and isolines for the flow past a semi-circular cylinder at $\operatorname{Re}=3000$ with $\tau=10 \% d$.

for all thicknesses: the control efficiency of case 5 is inversely proportional to the porous layer thickness $\tau$. Indeed, in Tables 3,4 and 5 one can clearly see that with $\tau=2.5 \% d$ the drag reduction achieved with case 5 , leads to $-15 \%$ for $\operatorname{Re}=550$ and $-10.5 \%$ for $\mathrm{Re}=3000$. This promising assessment seems to be due to the fact that the flow resistance inside a thin porous layer is less than a thick layer but the shedding is still well tuned thanks to the position of porous coatings in the body corners. 


\begin{tabular}{lllll}
\hline & \multicolumn{2}{c}{$\mathrm{Re}=550$} & \multicolumn{2}{c}{$\mathrm{Re}=3000$} \\
\hline$\tau=5 \% d$ & $F_{x}$ & Enstrophy & $F_{x}$ & Enstrophy \\
\hline case 0 (uncontrolled case) & 0.957 & 158.4 & 0.926 & 334.2 \\
case 1 & $0.774(-19 \%)$ & $123.7(-22 \%)$ & $0.999(+8 \%)$ & $285.2(-15 \%)$ \\
case 2 & $0.914(-4 \%)$ & $123.5(-22 \%)$ & $0.979(+6 \%)$ & $253.7(-24 \%)$ \\
case 3 & $0.751(-22 \%)$ & $110.4(-30 \%)$ & $0.947(+2 \%)$ & $247.6(-26 \%)$ \\
case 4 & $0.618(-35 \%)$ & $127.6(-19 \%)$ & $0.819(-12 \%)$ & $326.2(-2 \%)$ \\
case 5 & $0.753(-21 \%)$ & $135.4(-15 \%)$ & $0.847(-8.5 \%)$ & $312.3(-6.5 \%)$ \\
\hline
\end{tabular}

Table 4. Reduction effects brought by the different porous layer configurations in comparison to the uncontrolled case at $\mathrm{Re}=550$ and $\mathrm{Re}=3000$ with $\tau=5 \%$.

\begin{tabular}{lllll}
\hline & \multicolumn{2}{c}{$\mathrm{Re}=550$} & \multicolumn{2}{c}{$\mathrm{Re}=3000$} \\
\hline$\tau=2.5 \% d$ & $F_{x}$ & Enstrophy & $F_{x}$ & Enstrophy \\
\hline case 0 (uncontrolled case) & 0.957 & 158.4 & 0.926 & 334.2 \\
case 1 & $0.821(-14 \%)$ & $139.3(-12 \%)$ & $1.011(+9 \%)$ & $318.4(-5 \%)$ \\
case 2 & $0.879(-8 \%)$ & $134.5(-15 \%)$ & $1.018(+10 \%)$ & $283.7(-15 \%)$ \\
case 3 & $0.824(-14 \%)$ & $129.6(-18 \%)$ & $0.929(+0.4 \%)$ & $271.1(-19 \%)$ \\
case 4 & $0.768(-20 \%)$ & $140.2(-11 \%)$ & $0.806(-13 \%)$ & $331.4(-0.8 \%)$ \\
case 5 & $0.810(-15 \%)$ & $143.4(-10 \%)$ & $0.828(-10.5 \%)$ & $320.6(-4 \%)$ \\
\hline
\end{tabular}

Table 5. Reduction effects brought by the different porous layer configurations in comparison to the uncontrolled case at $\mathrm{Re}=550$ and $\operatorname{Re}=3000$ with $\tau=2.5 \% d$.

a)

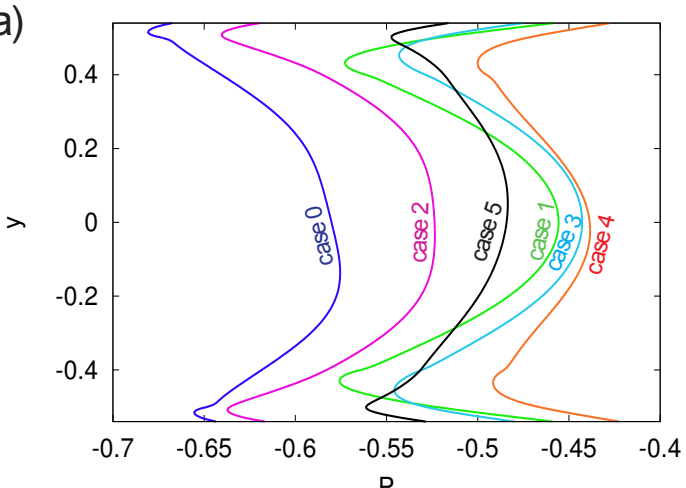

b)

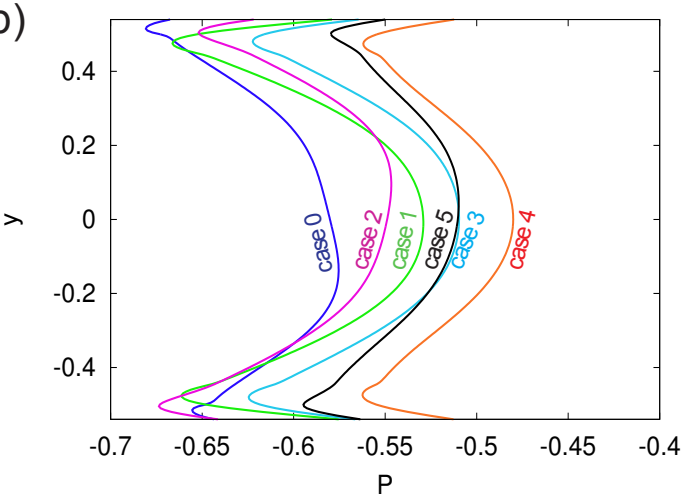

c)

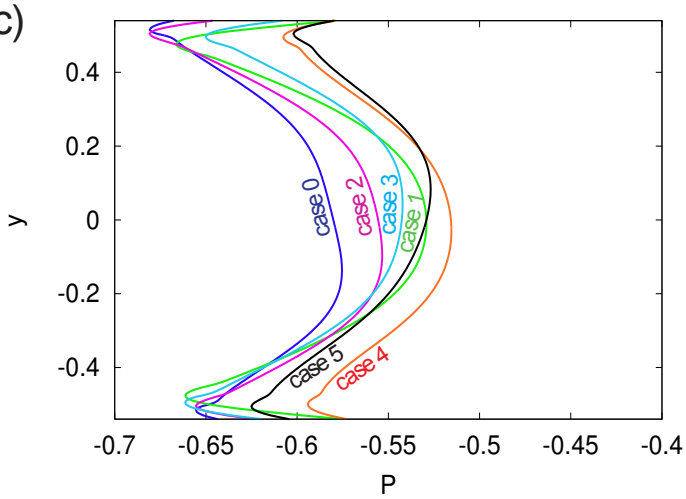

Fig. 15. Mean pressure profiles at the rear end of the body with a) $\tau=10 \% d$, b) $\tau=5 \% d$ and c) $\tau=2.5 \% d$ at $\operatorname{Re}=550$. 

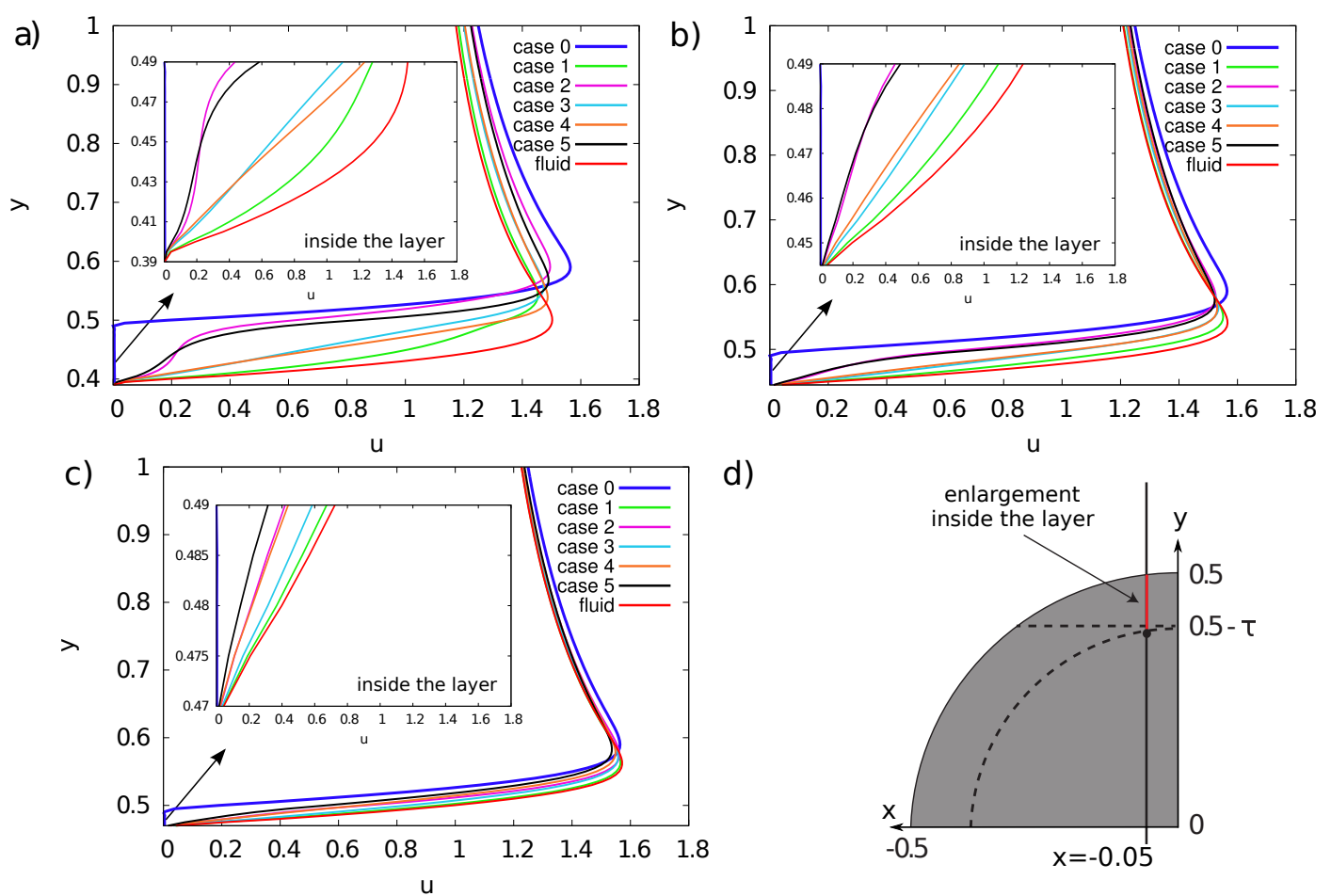

d)

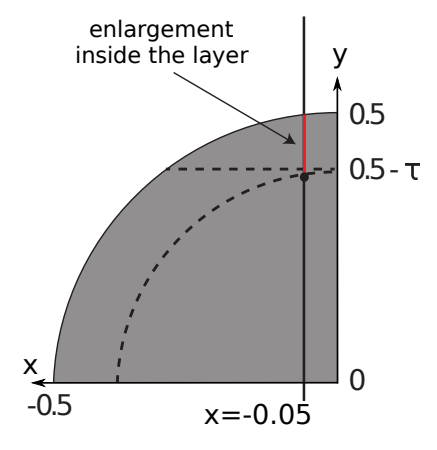

Fig. 16. Mean profiles of the streamwise component of the velocity $\mathrm{u}$ at $x=-0.05$ with a) $\tau=10 \% d$, b) $\tau=5 \% d$ and c) $\tau=2.5 \% d$ at $\mathrm{Re}=550$. d) Pattern of profile configuration at $x=-0.05$ showing the location of the profiles enlargement inside the layer proposed in $\mathrm{a}$ ), b) and $\mathrm{c}$ ).

a)

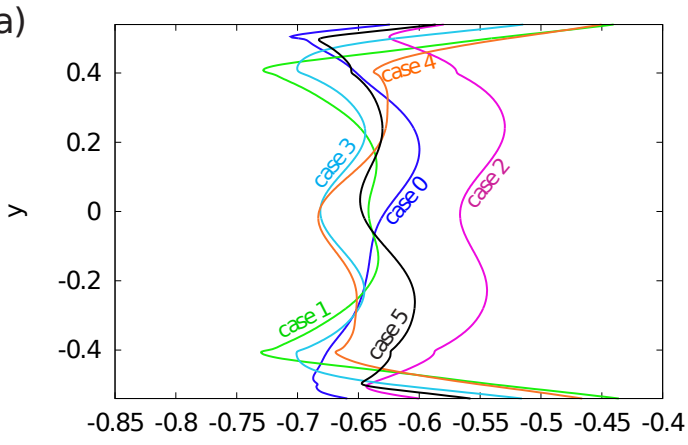

C)

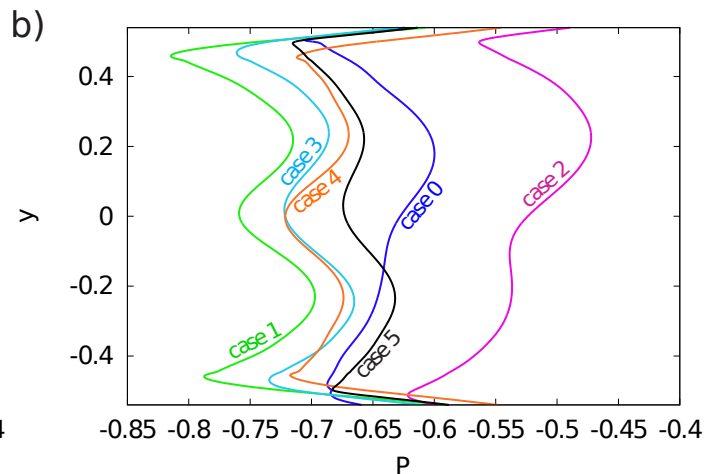

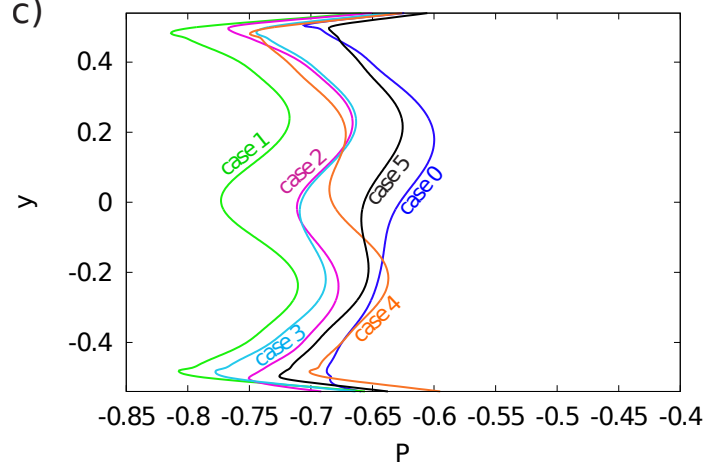

Fig. 17. Mean pressure profiles at the rear end of the body with a) $\tau=10 \% d$, b) $\tau=5 \% d$ and c) $\tau=2.5 \% d$ at $\operatorname{Re}=3000$. 

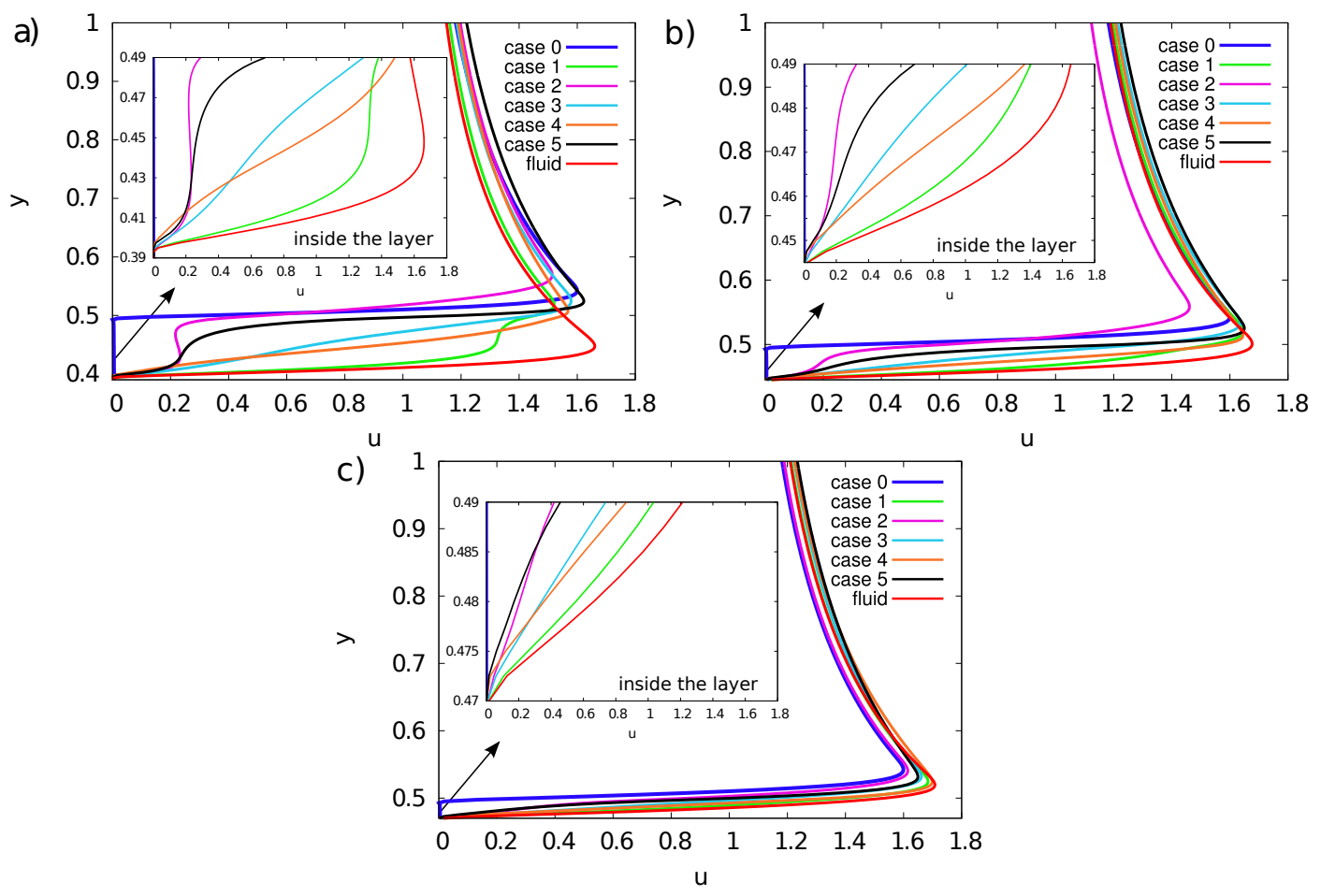

Fig. 18. Mean profiles of the streamwise component of the velocity $\mathrm{u}$ at $x=-0.05$ with a) $\tau=10 \% d$, b) $\tau=5 \% d$ and c) $\tau=2.5 \% d$ at $\mathrm{Re}=3000$ (cf Fig. 16 d) for profiles location.) 


\section{CONCLUSION}

In this work a passive control technique with porous coatings was implemented around a semi-circular cylinder in order to regularize flow and reduce drag forces for laminar and transitional flows. The direct numerical simulations were performed using vortex-penalization method which allows to compute simultaneously the three fluid-porous-solid media. In the numerical study, the effect of the porous layer permeability on the control results was first analyzed. Highly permeable media were more efficient, as expected. Then, several configurations of porous devices were compared. The results showed that introducing porous layers only at the top and bottom of the solid body permits to better manipulate the vortex shedding and to achieve relevant control performances. A further study on the influence of the porous layer thickness on the control efficiency was performed. It showed that a thin layer with intermediate permeability, introduced in both edges of the back wall, generates a significant drag reduction. As the ultimate target of this work is the drag reduction related to side mirrors, this type of configuration would be easy to implement industrially and at low cost, since it only consists in adding an intermediate-porous ring on the edge wall of the mirror. Although the present work needs to be complemented by threedimensional studies to confirm the quantitative control trends that were observed in the $2 \mathrm{D}$ experiments, it thus opens interesting perspectives towards efficient drag control devices.

\section{References}

[1] Rouméas, M., Gilliéron, P., and Kourta, A., 2009. "Analysis and Control of the Near-Wake Flow Over a Square-Back Geometry". Computers \& Fluids, 38, pp. 60-70.

[2] Bruneau, C. H., Creusé, E., Depeyras, D., Gilliéron, P., and Mortazavi, I., 2010. "Coupling Active and Passive Techniques to Control the Flow Past the Square Back Ahmed Body". Computers \& Fluids, 38, pp. 1875-1892.

[3] Bruneau, C. H., and Mortazavi, I., 2004. "Passive Control of the Flow Around a Square Cylinder Using Porous Media". International Journal for Numerical Methods in Fluids, 46, pp. 415-433.

[4] Bruneau, C. H., and Mortazavi, I., 2006. "Control of Vortex Shedding Around a Pipe Section Using a Porous Sheat". Journal of Offshore and Polar Engineering, 16.

[5] Bruneau, C. H., Gilliéron, P., and Mortazavi, I., 2007. "Flow Manipulation Around the Ahmed Body With a Rear Window Using Passive Strategies". Comptes Rendus Mecanique, 335.

[6] Bruneau, C. H., and Mortazavi, I., 2008. "Numerical Modelling and Passive Flow Control Using Porous Media". Computers \& Fluids, 37, pp. 488-498.

[7] Bruneau, C. H., Gilliéron, P., and Mortazavi, I., 2008. "Passive Control Around a Two-Dimensional Square Back Ahmed Body Using Porous Media". Journal of Fluids Engineering, 130.

[8] Carbou, G., 2008. "Brinkmann Model and Double Penalization Method for the Flow Around a Porous Thin Layer". Journal of Mathematical Fluid Mechanics, 10, pp. 126-158.

[9] Angot, P., Bruneau, C. H., and Fabrie, P., 1999. "A Penalization Method to Take Into Account Obstacles in Incompressible Viscous Flows". Numerische Mathematik, 81, pp. 497-520.

[10] Coquerelle, M., and Cottet, G. H., 2008. "A Vortex Level Set Method for the Two-Way Coupling of an Incompressible Fluid with Collinding Rigid Bodies". Journal of Computational Physics, 227, pp. 9121-9137.

[11] Rossinelli, D., M.Bergdorf, Cottet, G.-H., and Koumoutsakos, P., 2010. "Gpu accelerated simulations of bluff body flows using vortex particle methods". Journal of Computational Physics.

[12] Cottet, G. H., Gallizio, F., Magni, A., and Mortazavi, I., 2011. "A Vortex Penalization Method for Flows with Moving Immersed Obstacles". In Proceedings of ASME-JSME-KSME Joint Fluids Engineering Conference, July, 2011, ASME, pp. 3703-3708. Paper number AJK2011-20015.

[13] Gazzola, M., van Rees, W., and Koumoutsakos, P., 2012. "C-start: optimal start of larval fish". Journal of Fluid Mechanics, 698, pp. 5-18.

[14] van Rees, W., Gazzola, M., and Koumoutsakos, P., 2013. "Optimal shapes for anguilliform swimmers at intermediate reynolds numbers". Journal of Fluid Mechanics, 722.

[15] Cottet, G. H., and Koumoutsakos, P. D., 2000. Vortex Methods - Theory and Practice. Cambridge University Press.

[16] Koumoutsakos, P., and Leonard, A., 1995. "High-resolution simulations of the flow around an impulsively started cylinder using vortex methods". Journal of Fluid Mechanics, 296, pp. 1-38.

[17] Cottet, G. H., and Maitre, E., 2004. "A Level-Set Formulation of Immersed Boundary Methods for Fluid-Structure Interaction Problems". Comptes Rendus de l'Acadmie des Sciences de Paris, 338, pp. 51-56.

[18] Chorin, A. J., 1974. "Numerical Study of Slighly Viscous Flow". Journal of Fluid Mechanics, 57, pp. 785-796.

[19] Ghoniem, A. F., and Gagnon, Y., 1987. "Vortex Simulation of Laminar Recirculating Flows". Journal of Computational Physics, 68, pp. 346-377.

[20] Sethian, J. A., and Ghoniem, A. F., 1988. "Validation Study of Vortex Methods". Journal of Computational Physics, 74, pp. 283-317.

[21] Creusé, E., Giovannini, A., and Mortazavi, I., 2009. "Vortex Simulation of Active Control Strategies for Transitional Backward-Facing Step Flows". Computers \& Fluids, 38, pp. 1348-1360. 
[22] Monaghan, J. J., 1985. “Extrapolating b-Splines for Interpolation”. Journal of Computational Physics, 60, pp. $253-262$.

[23] Chatelain, P., and Koumoutsakos, P., 2010. "A Fourier-Based Elliptic Solver For Vortical Flows With Periodic and Unbounded Directions". Journal of Computational Physics, 229, pp. 2425-31.

[24] Cottet, G. H., Gallizio, F., Magni, A., and Mortazavi, I., 2010. “A Vortex Immersed Boundary Method for Bluff Body Flows". In Proceedings of 3rd Joint US-European ASME Fluids Engineering Summer Metting, August, 2010, ASME, pp. 2409-2414. Paper number FEDSM-ICNMM2010-30787.

[25] Beavers, G. D., and Joseph, D. D., 1967. "Boundary Conditons at a Naturally Permeable Wall". Journal of Fluid Mechanics, 30, pp. 197-207.

[26] Breugem, W. P., Boersma, B. J., and Uittenbogaard, R. E., 2005. “The Laminar Boundary Layer Over a Permeable Wall". Transport in Porous Media, 59, pp. 267-300.

[27] Hanspal, N. S., Waghode, A. N., Nassehi, V., and Wakeman, R. J., 2006. "Numerical Analysis of Coupled Stokes/Darcy Flows in Industrial Filtrations". Transport in Porous Media, 64, pp. 73-101.

[28] Nield, D. A., and Bejan, A., 1999. Convection in Porous Media. Springer.

[29] Farhadi, M., Sedighi, K., and Fattahi, E., 2010. "Effect of a Splitter Plate on Flow Over a Semi-Circular Cylinder". In Proceedings of the Institution of Mechanical Engineers, Part G: Journal of Aerospace Engineering, pp. $224-321$.

[30] Boisaubert, N., and Texier, A., 1998. "Effect of a Splitter Plate on the Near-Wake Development of a Semi-Circular Cylinder.". Experimental Thermal and Fluid Science, 16, pp. 100-111.

[31] Noca, F., Shiels, D., and Jeon, D., 1999. "A Comparison of Methods for Evaluating Time-Dependent Fluid Dynamic Forces on Bodies, Using Only Velocity Fields and Their Derivatives”. Journal of Fluids and Structures, 13, pp. 551578.

[32] Ploumhans, P., and Winckelmans, G. S., 2000. "Vortex methods for high-resolution simulations of viscous flow past bluff bodies of general geometry". pp. 354-406. 